\title{
Diagnosing spin at the LHC via vector boson fusion
}

\author{
Matthew R. Buckley ${ }^{a, b}$ and Michael J. Ramsey-Musolf ${ }^{c, a}$ \\ ${ }^{a}$ Department of Physics, California Institute of Technology, \\ 1200 California Ave, Pasadena, CA 91125, U.S.A. \\ ${ }^{b}$ Center for Particle Astrophysics, Fermi National Accelerator Laboratory, \\ P.O. Box 500 Batavia, IL 60510, U.S.A. \\ ${ }^{c}$ Department of Physics, University of Wisconsin-Madison, \\ 1150 University Avenue, Madison, WI 53706, U.S.A. \\ E-mail: mbuckley@fnal.gov, mjrm@physics.wisc.edu
}

\begin{abstract}
We propose a new technique for determining the spin of new massive particles that might be discovered at the Large Hadron Collider. The method relies on pairproduction of the new particles in a kinematic regime where the vector boson fusion production of color singlets is enhanced. For this regime, we show that the distribution of the leading jets as a function of their relative azimuthal angle can be used to distinguish spin-0 from spin- $\frac{1}{2}$ particles. We illustrate this effect by considering the particular cases of (i) strongly-interacting, stable particles and (ii) supersymmetric particles carrying color charge. We argue that this method should be applicable in a wide range of new physics scenarios.
\end{abstract}

Keywords: Jets, Hadronic Colliders

ArXiv EPRINT: 1008.5151 


\section{Contents}

1 Introduction 1

2 Analytic calculations with Abelian examples 4

$\begin{array}{lll}3 & \text { Simulations with QCD gauge forces } & 7\end{array}$

4 Conclusion $\quad 10$

\section{Introduction}

The $\mathrm{TeV}$ energy scale is expected to contain an array of new particles predicted in extensions of the Standard Model (SM) that address electroweak symmetry breaking, naturalness, and hierarchy problems. Among the most widely discussed scenarios are supersymmetry, extra dimensions, grand unified theories, and technicolor. Assuming that new particles are discovered, the challenge will be to ascertain which new physics model - including the possibility of one not yet considered - best accounts for them. This task will be complicated by a degeneracy among many scenarios in basic experimental signatures. A determination of the particles' quantum numbers will provide key input in discriminating among the candidate models.

One of the most important measurements - and the focus of this paper — is the determination of particle spin. It is, for example, one of the primary ways to distinguish supersymmetry from universal extra dimensions (see e.g. [1]). Theorists have devoted considerable effort to developing methods for diagnosing spin at a hadron collider or a possible new linear collider [2-8]. The simplest method is to determine the kinematic dependence of the Drell-Yan pair production cross section [4]. More sophisticated methods rely on the spindependence encoded in the decays of the produced particles. This approach is challenging for $p p$ collisions at the Large Hadron Collider (LHC) since a full kinematic reconstruction of the decay is not possible in events with two or more invisible particles, barring long decay chains where the origin of the visible particles can be unambiguously identified. As many extensions of the SM contain dark matter candidates that would be produced in cascades at the LHC, this can be an especially limiting constraint. Though it can be quite powerful in many circumstances $[2,3,5-7]$, there are many generic examples of new physics where such technique cannot be applied. Similar considerations apply to the measurement of spin information contained in quantum interference between different decay amplitudes [8], though this measurement technique is "model independent," there are many cases where insufficient kinematic information is available to reconstruct the observables which contain spin information. Though each of the spin measurement techniques referenced here have many areas of applicability, none can be used in every new physics scenario. It is therefore 


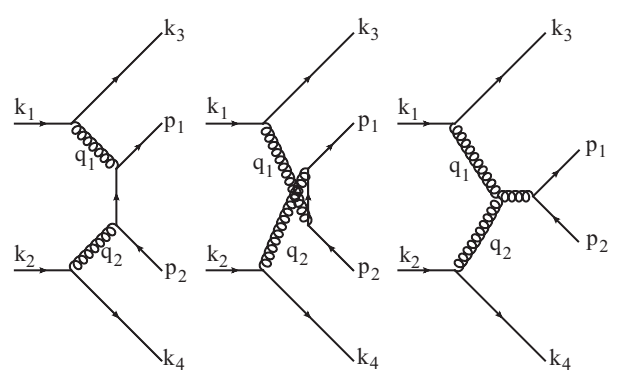

Figure 1. Representative Feynman diagrams which dominate the production cross section after application of VBF cuts, as described in text. In the abelian calculation, only the first two ( $t$ channel) diagrams exist. The massive new physics particles have momenta $p_{1}$ and $p_{2}$; though drawn as fermions, we will also consider scalars in this work. The incoming and outgoing partons with momenta $k_{1}, k_{2}, k_{3}$ and $k_{4}$ are drawn as quarks, but in our work, we also consider antiquark and gluon contributions. Momentum labels are the same as in figure 3.

desirable to develop complementary spin probes that may either be applied in cases where the known techniques fail or used to confirm spin determinations made by other methods.

In this paper, we propose a new spin measurement technique that does not require even partial reconstruction of the new particles' momenta. The method relies on isolating a kinematic regime in which vector boson fusion (VBF) pair production is enhanced relative to other mechanisms (e.g., Drell-Yan). Representative Feynman diagrams with the largest contributions after these cuts are shown in figure 1. Either of the initial state partons (labeled $k_{1}$ and $k_{2}$ ) can be a quark, anti-quark, or gluon, and the partonic identity of $k_{1}$ and $k_{2}$ need not be the same. In this regime, we focus on the forward (or leading) jets associated with the final state partons labeled $k_{3}$ and $k_{4}$ and show that their differential distribution as a function relative jet-jet azimuthal angle, $\Delta \phi$, can be used to diagnose the spin-statistics of the produced massive particles.

In particular, we find that the sign of the $\cos 2 \Delta \phi$ term in the distribution differs for spin-0 and spin- $1 / 2$ particles. The dependence on $\Delta \phi$ can be understood as the physical observable arising from the interference of helicity amplitudes associated with the vector bosons involved in the pair production of new particles. Information on the new particles' spin - encoded in the Lorentz structure of their pair production amplitude - is communicated to the interfering vector boson helicity amplitudes. The resulting azimuthal distribution of the leading jets probes this information. As we show below, by experimentally determining the sign of the $\cos 2 \Delta \phi$ component of the dijet distribution, one has as a result particularly clean "diagnostic" of the new particle's spin.

Our investigation of this technique has been heavily influenced by previous studies of dijet correlations in VBF [9-13]. The earliest work concentrated on the use of weak boson fusion (WBF) to discover an invisibly decaying Higgs boson or to probe the CP properties of the Higgs coupling to the weak bosons [9, 10]. At the partonic level, the WBF process can be isolated from the large QCD and $t \bar{t}$ dijet backgrounds using the distinctive pseudorapidity difference $(\Delta \eta)$ and dijet invariant mass $\left(m_{j j}\right)$ distributions. The resulting signal associated with the invisibly decaying Higgs involves an enhancement of the differen- 
tial cross section for $\Delta \phi<\pi / 2$, whereas the remaining Standard Model dijet background favors the opposite hemisphere. Subsequent Monte Carlo studies by the ATLAS collaboration indicate a persistence of this signal after accounting for parton showering and various trigger options [14]. A discovery of the invisibly decaying Higgs would be possible with 30 $\mathrm{fb}^{-1}$ of integrated luminosity for a wide range or Higgs boson masses if the effective cross section is more than $60 \%$ of the SM Higgs production cross section.

Subsequent studies of the Higgs boson CP properties considered both WBF [10] and gluon fusion (GF) $[11,12]$. The $\Delta \eta$ and $m_{j j}$ distributions for the two classes of VBF processes are quite distinct, leading to different event selection criteria for each. For both processes, however, the appearance of a distinctive $\Delta \phi$ distribution associated with various CPeven and CP-odd couplings of the Higgs to vector bosons appears to be associated with leading jets that are widely separated in pseudorapidity (see, e.g., figure 11 of ref. [12]). Thus, imposing an event selection cut on $|\Delta \eta|$ appears advantageous for enhancing the $\Delta \phi$ signal, even for GF for which the $\Delta \eta$ distribution itself is not useful for isolating the fusion process.

Motivated by these observations, we consider the $\Delta \phi$ distributions for pair production of new particles through the VBF process in the large $\Delta \eta$ regime. We find that as the $\Delta \eta$ cut is relaxed, the distinctive signal associated with spin- 0 or spin- $1 / 2$ particles vanishes. To gain further insight into this result, we show analytically that the signal is associated with the two fusion bosons in the color (or weak isospin) singlet state. In the case of GF, color singlet dominance - or color "coherence" of the leading jets - has been intuitively associated with a rapidity gap, since a color singlet object is unlikely to radiate significantly in the central region (for a discussion of these expectations, see, e.g. ref. [15] and references therein). Overlapping minimum bias events and minijet activity will populate the central region - considerations that are likely to apply to the earlier GF studies [11, 12] as well as in the present case. Nevertheless, it is intriguing that the spin-dependent signal associated with the underlying hard event is associated with both a large separation in pseudorapidity and a color singlet configuration.

As a practical matter, one must be able to distinguish the leading jets from jets produced in the decays of the produced particle pair, particularly in the case of GF where the latter is produced strongly. In this respect, we note that the forward jets relevant for our observable are essentially initial state radiation (ISR) associated with the hard event. Typically, ISR is an undesirable but unavoidable fact of life at hadronic colliders, ISR jets being easily confused with jets originating in decays of new physics. Considerable work has gone into separating these two classes of hadronic activity (see e.g. ref. [16, 17], in which ISR must be distinguished from the decays of gluinos in order to make mass measurements, and ref. [18] where jet kinematics are used to statistically identify ISR jets). These studies suggest that the prospects are promising for isolating the jets of interest to the spin determination from the decay products. We defer a detailed study of jet identification (i.e. hadronization, jet finding, and inclusion of full ISR and FSR effects) to future work, and concentrate here on the physics of the underlying signal. While these effects are nontrivial, we note that the more complete analysis on the VBF Higgs search [14] — from which we draw motivation - does include these effects, and finds that the signal in $\Delta \phi$ survives. 
In what follows, we first demonstrate the spin-dependent $\Delta \phi$ correlation with a simulation for scalar and spinor stable strongly-interacting particles, i.e. $R$-hadrons $[19,20]$. This is an interesting illustrative scenario in its own right, as the spin of stable particles cannot be measured using the standard techniques (see refs. [21, 22] for exceptions), but also has the attractive property of being background-free. We also show, through explicit simulations, that the same effect is present for strongly interacting supersymmetric particles and for Standard Model $t \bar{t}+j j$ production. The fact that this spin measurement technique survives in the supersymmetric example is of particular interest, not only because of the wide body of literature on this type of new physics. Supersymmetric gluinos are Majorana, and it is useful to see that the spin correlation is present in this case as well as for Dirac fermions. Furthermore, the supersymmetric models have a large number of VBF production diagrams (beyond those present in the simplified $R$-hadron model), and it is again instructive to see that the spin-correlation remains after their inclusion. Note that, in all these cases, the relevant vector boson is the gluon. We anticipate that the same spin-dependent $\Delta \phi$ signal should also be present for weak boson fusion (WBF). Although WBF can be isolated through the $\Delta \eta$ and $m_{j j}$ distributions, and backgrounds further reduced through use of the central jet veto (see, e.g., refs. [23-26] and references therein), the event rate would be very reduced. Consequently, we concentrate here on the GF case in order to demonstrate the basic concept of the technique.

In the next section we explain in detail the underlying concept behind our new spin measurement technique, and analytically demonstrate its utility in the toy examples of scalars and spinors charged under an abelian gauge group. In section 3, we apply the technique to simples models with non-abelian QCD interactions, using MadGraph/MadEvent [27] and CalcHEP simulations [28]. As will be shown, inclusion of color factors greatly complicates the analytic calculation, but does not change the overall applicability of the method.

\section{Analytic calculations with Abelian examples}

As outlined in the Introduction, we are interested in the pair production via VBF of two heavy particles with two forward jets. The interference of the vector bosons helicities is set by the Lorentz structure of the pair production matrix element, and in turn affects the distribution in $\Delta \phi$ of the two forward jets. To make this causal chain clear, we begin with an abelian gauge group as the vector bosons which fuse, and consider only the dominant Feynman diagrams in order to make the computation tractable (see figure 1). The full calculation of abelian gauge groups, kinematic cuts, and all involved diagrams are necessary, but require numeric simulation to calculate, and will be described in section 3 . Here, we restrict ourselves to a tractable subset of diagrams and significantly constrained kinematics, in order to gain some intuition for the expected signal.

To set the theoretical framework, we consider pair production of massive particles in a regime where VBF is kinematically favored. As illustrated in figure 2, the event contains two forward jets $\left(J_{1}\right.$ and $\left.J_{2}\right)$ as well as the two new particles (each having mass $m$ and momenta $p_{1}$ and $p_{2}$ ). The azimuthal angle $\phi_{j}$ of each jet is defined relative to a 


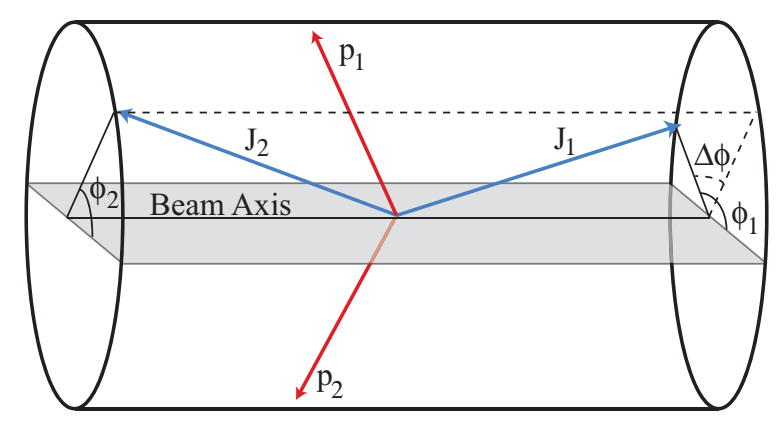

Figure 2. A VBF event in an idealized detector volume. The massive new physics particles are labeled $p_{1}$ and $p_{2}$; their momentum is not required to be reconstructed. The two forward jets $J_{1}$ and $J_{2}$ - numeric ordering here is arbitrary — have azimuthal angles $\phi_{1}$ and $\phi_{2}$, which measured from an arbitrary plane. Only the difference in angle $\Delta \phi \equiv\left|\phi_{1}-\phi_{2}\right|$ has physical meaning.

reference plane. The location of the reference plane is arbitrary, as is direction of the $z$-axis, making the labeling of jets as " 1 " and "2" arbitrary as well (in the absence of jet tagging). Consequently, the only physically quantity is the relative azimuthal angle $\Delta \phi$.

Our labeling of the kinematics of the VBF amplitude is indicated in figure 3, largely following the conventions of ref. [13]. Frames I and II are the Breit frames for $q_{1}$ and $q_{2}$, respectively; i.e. the momenta $q_{1,2} \equiv\left(0,0,0, Q_{1,2}\right)$. Frame $\mathrm{X}$ is the center of mass frame for the pair production of the new physics particles. Again, though we draw all diagrams as if the incoming and outgoing partons are quarks, in our full numerical calculations, we do include antiquark and gluon contributions. The polarization vectors of the gauge bosons are proportional to $e^{i h_{i} \phi_{i}}$, where $i=1,2$, and $h_{i}$ is the polarization of the boson defined relative to the positive $z$-axis. As we are interested in the kinematic region where $k_{3}$ and $k_{4}$ are very forward, for our analytic calculation we can make the approximation that these particles are colinear with the $z$-axis (i.e. colinear with $q_{1}$ and $q_{2}$ ). With this choice, the phase angles $\phi_{1}$ and $\phi_{2}$ are the same as the physical azimuthal angles in figure 2. Clearly, in the full calculation, this is not a completely valid approximation; but as we shall see in the numeric calculations, the conclusions we derive here do apply, as the kinematics cuts which select VBF-type production also force the jets to be approximately colinear.

With these choices of angles, the matrix elements for the emissions of the gauge bosons can be expressed as $\mathcal{M}_{i}\left(\theta_{i}, \phi_{i}, h_{i}\right)=e^{i h_{i} \phi_{i}} \mathcal{M}_{i}\left(\theta_{i}, 0, h_{i}\right)$. Thus, any dependence on $\phi_{1}$ and $\phi_{2}$ in the amplitude must be due to interference between matrix elements of the different polarizations. The resulting contribution to the differential cross section then a yields set of 24 functions (sines and cosines) whose arguments are linear combinations of the $\phi_{j}$. As discussed above, in this paper we retain only the terms dependent on $\Delta \phi$. Moreover, if the pair production amplitude is CP-invariant as we will assume here, then only the cosine terms survive. Thus,

$$
\frac{d \sigma}{d \Delta \phi}=A_{0}+A_{1} \cos \Delta \phi+A_{2} \cos 2 \Delta \phi
$$

Depending on the properties of the new physics, it may be possible to construct a physically relevant plane in each event, which would allow unambiguous definition of 




Figure 3. Kinematics for VBF in Frames I, II and X. Frames I and II are the Breit frames for the bosons $q_{1}$ and $q_{2}$, and Frame $\mathrm{X}$ is the center of mass frame for the pair production of $p_{1}$ and $p_{2}$. See text for further details. Azimuthal angles $\phi_{1}$ and $\phi_{2}$ are defined relative to the production plane of the massive particles (momenta $p_{1}$ and $p_{2}$ ). Polar angles $\theta_{1}, \theta_{2}$ and $\Theta$ are defined relative to the beam axis in their respective frames.

$\phi_{1}+\phi_{2}$. In such cases, the coefficients of the remaining functions may contain additional interesting information about the properties of the new physics. However, as this is not universally possible in all examples, for this work, we concentrate only on the $\Delta \phi$ terms.

The VBF kinematics induces a basic $\cos \Delta \phi$ dependence on the cross section that typically overshadows the contribution to $A_{1}$ from the interfering amplitudes [9]. The fusion process tends to knock the produced pair and recoiling jets on opposite sides of a suitably defined reference plane, favoring a smaller $\Delta \phi$. In resonant production, this $\Delta \phi$ bias can be exploited in searches for "invisibly" decaying Higgs bosons produced through WBF $[9,14]$. In contrast, the $\cos 2 \Delta \phi$ term is dominated by the interference of the VBF amplitudes, receiving no significant kinematic contribution. Importantly, we find that the sign of the coefficient function $A_{2}$ is opposite for scalars (positive $A_{2}$ ) and spinors (negative $A_{2}$ ). While in this section, we only demonstrate the sign flip between the two spin choices, the results of our simulations in section 3 will show that, while the $\cos 2 \Delta \phi$ correlation is subleading, it is sufficiently large to make its relative sign a useful spin diagnostic.

The coefficient $A_{2}$ arises from the interference of the VBF pair production matrix elements $\mathcal{M}_{\text {pair }}$ that differ by two unit of boson polarization:

$$
A_{2}=(\mathcal{P S}) \sum_{\substack{h_{1}, h_{1}^{\prime}, h_{2}, h_{2}^{\prime} \\\left|h_{i}-h_{i}^{\prime}\right|=2}}\left(\mathcal{M}_{1}\left(h_{1}\right) \mathcal{M}_{2}\left(h_{2}\right) \mathcal{M}_{\text {pair }}\left(h_{1}, h_{2}\right)\right)\left(\mathcal{M}_{1}\left(h_{1}^{\prime}\right) \mathcal{M}_{2}\left(h_{2}^{\prime}\right) \mathcal{M}_{\text {pair }}\left(h_{1}^{\prime}, h_{2}^{\prime}\right)\right)^{*}
$$

Here $(\mathcal{P S})$ is the phase space factor; $\mathcal{M}_{1}$ and $\mathcal{M}_{2}$ are the parton $\rightarrow$ parton + gauge boson production amplitudes (see figure 3 ); and $\mathcal{M}_{\text {pair }}$ is the VBF amplitude for production of the heavy pair. Performing the helicity sums leads to

$$
A_{2}=(\mathcal{P S}) \mathcal{M}_{1}(+1) \mathcal{M}_{1}(-1)^{*} \mathcal{M}_{2}(-1) \mathcal{M}_{2}(+1)^{*} \mathcal{M}_{\text {pair }}(+1,-1) \mathcal{M}_{\text {pair }}(-1,+1)^{*}+(1 \leftrightarrow-1) .
$$

In the illustrative simple case considered in this section, that of abelian gauge bosons (e.g., photons), the fusion amplitude $\mathcal{M}_{\text {pair }}$ is generated by the standard Compton diagrams (with gauge coupling $g$ ). For spin-0 particles of charge $Q$, a straightforward computation 
of the $t$-channel diagrams of figure 1 gives

$$
\mathcal{M}_{\text {scalar }}=g^{2} Q^{2}\left\{\left(\epsilon_{1} \cdot \epsilon_{2}\right)-4\left[\frac{\left(p_{1} \cdot \epsilon_{1}\right)\left(p_{1}-q_{1}\right) \cdot \epsilon_{2}}{q_{1}^{2}-2 p_{1} \cdot q_{1}}+\frac{\left(p_{1} \cdot \epsilon_{2}\right)\left(p_{1}-q_{2}\right) \cdot \epsilon_{1}}{q_{2}^{2}-2 p_{1} \cdot q_{2}}\right]\right\} .
$$

Choosing polarization vectors (with $h_{i}=-1,0,+1$ ) to reflect the angular momentum in the $z$-direction, $\epsilon_{1}^{ \pm}=\epsilon_{2}^{ \pm} \equiv \epsilon^{ \pm}$. It is then obvious that $\mathcal{M}(+1,-1)=\mathcal{M}(-1,+1)$, which demonstrates that

$$
A_{2} \propto\left|\mathcal{M}_{\text {scalar }}( \pm 1, \mp 1)\right|^{2},
$$

and is therefore positive. Though a more lengthly calculation is involved, this argument also holds for VBF scalar pair production by non-abelian gauge bosons.

The calculation for spinors is similarly straightforward, but the origin of the negative sign of $A_{2}$ (opposite that of the scalars) is less transparent. However, a closed form expression can be obtained in the limit that the two abelian gauge bosons are massless and on-shell $\left(q_{1}^{2}=q_{1}^{2}=0\right)$. Defining $\lambda=\sqrt{1-4 m^{2} / s}$ where $s=\left(k_{1}+k_{2}\right)^{2}$, we find

$$
A_{2} \propto-\frac{64 m^{2}}{s}\left(1+\frac{4 m^{2}}{s \lambda} \tanh ^{-1} \lambda\right)
$$

which is clearly negative. It is interesting to note that the minus sign (relative to the scalar calculation), arises from the anticommutation of fermion operators in the simplification of the expression for $\mathcal{M}_{\text {pair }}(+1,-1) \mathcal{M}_{\text {pair }}(-1,+1)^{*}$, thus making the connection between the sign of $\cos 2 \Delta \phi$ and spin clear.

We now briefly mention the difficulties in analytic calculation for the non-abelian fermionic example, before continuing on to the numeric simulations. In the explicit calculation of the non-abelian example, two terms arise: a positive contribution with an antisymmetric color factor $\left[T^{a}, T^{b}\right]$, and a negative contribution - identical to eq. (2.6) up to an overall a symmetric color factor $\left\{T^{a}, T^{b}\right\}$. These two terms correspond to the two gluons being in a color octet and color singlet state, respectively. As we shall see, numeric calculation for off-shell gauge bosons confirms that the coefficient is overall negative, meaning that the color singlet state (symmetric term) dominates. This is an intriguing result, and we give a heuristic explanation. Helicity conservation and the kinematics of nearly forward jets (typical of ISR) favors a spatially symmetric configuration for the fusing gluons, and thus a color symmetric (singlet) two gluon state. However, without an application of kinematic cuts on the 4-body phase space, this is not reflected in the analytic calculation.

\section{Simulations with QCD gauge forces}

We therefore turn to a full calculation of the jet distributions using non-abelian gauge boson. As outlined previously, a realistic computation involves additional subtleties, though we find from simulations that the sign difference persists. The non-abelian Compton amplitude $\mathcal{M}_{\text {pair }}$ contains a dependence on the structure constants and generators of the gauge group as well as contributions from a diagram involving fusion of two gauge bosons to a third, followed by production of the heavy pair. In addition, the coefficients 
$A_{0}, A_{1}$ and $A_{2}$ receive additional contributions from heavy particle pair production via a bremsstrahlung gluon. If production was primarily via an on-shell intermediary (i.e., a resonance), the $\Delta \phi$ signal would be governed by the coupling of the intermediary to the two gauge bosons rather than by the spin of the produced pair. However, such production is not dominant in the cases we consider here.

To illustrate the spin-dependent sign of the $\cos 2 \Delta \phi$, we first specialize to VBF production of $R$-hadron pairs at the LHC (for an overview and references, see e.g., ref. [29]). These stable, strongly interacting particles would have striking signatures at the LHC, either appearing as "slow muons" that are highly ionizing [29, 30], or as stopped tracks in the detector [31,32]. The background is therefore limited to detector errors, which we will ignore for this study. $R$-hadrons were originally proposed in supersymmetric models with a gluino or squark as the lightest (or next-to-lightest) supersymmetric particle [33-39], though other theories can provide similar particles (for an excellent overview, see ref. [29]). Null results from searches from ALEPH [40], CDF [41], and LEP2 [42], exclude particles of masses less than $\sim 200-250 \mathrm{GeV}$, depending on the theoretical assumptions made, while the LHC should be able to find $R$-hadrons up to a few $\mathrm{TeV}$ in mass [29, 43]. In addition, since standard spin measurements cannot be applied to stable particles, new techniques would be necessary in order to study the spin of $R$-hadrons [22].

We assume some new $\mathbb{Z}_{2}$ quantum number ( $R$-parity in supersymmetry) that requires $R$-hadrons to be pair produced. As strongly interacting particles, this production can proceed via VBF mediated by gluons ("gluon fusion," or "GF"). Matrix elements for two heavy fermions or scalars plus two jets from $p p$ initial states are generated in MadGraph, and Monte Carlo simulations in MadEvent [27]; such simulations correctly include all interference terms as well as production from a bremsstrahlung gluon. To isolate the kinematics in which the GF process is enhanced relative to Drell-Yan, we impose the following cuts:

$$
\begin{aligned}
& \eta_{j_{1}} \cdot \eta_{j_{2}}<0, \\
& \left|\eta_{j}\right| \leq 5, \\
& \left|\eta_{j_{1}}-\eta_{j_{2}}\right| \geq 4.2 \\
& p_{T, j_{1}} \geq 30 \mathrm{GeV}, \\
& p_{T, j} \geq 20 \mathrm{GeV} \text {, } \\
& M_{j j} \geq 500 \mathrm{GeV} \\
& \left|\eta_{R-\text { hadron }}\right|<2.1 \text {, } \\
& p_{T, R \text {-hadron }}>50 \mathrm{GeV} \text {. }
\end{aligned}
$$

Here, $\eta_{j}$ is the jet pseudo-rapiditiy; $M_{j j}$ is the dijet invariant mass; and $p_{T}$ denotes the transverse momentum of a given particle. The jet cuts are necessary in order to reduce the non-VBF events [44, 45], while the $R$-hadrons cuts are of lesser importance, and simply require the heavy states appear in the barrel of the detectors.

We generate 40,000 events for the LHC at $\sqrt{s}=10 \mathrm{TeV}$ for simple, $500 \mathrm{GeV}$, scalar and Dirac spinor $R$-hadrons, in which the heavy particles only couple to gluons. After all cuts the spinor cross sections are $33 \mathrm{fb}$ (spinor) and $21 \mathrm{fb}$ (scalar). The differential cross sections $d \sigma / d \Delta \phi$ for the two models are shown in figure 4 , along with the best fits to eq. (2.1).

It has been demonstrated that the VBF-isolating jet cuts outlined in eq. (3.1) tend to induce a positive $\cos \Delta \phi$ mode in the distribution [9]. The expected positive $A_{1}$ mode is found both the scalar and spinor simulations. The $\cos 2 \Delta \phi$ coefficient $A_{2}$ is not affected by the cuts, and we see a significant difference between the two spin cases. The scalar case, as predicted, has a positive coefficient, while the spinor has a negative one. 




Figure 4. Differential cross section $d \sigma / d \Delta \phi$ as a function of difference in the azimuthal angle of VBF jets $\Delta \phi$. The differential cross section for fermionic R-hadron pair production is shown in blue, and the best fit to $A_{0}+A_{1} \cos \Delta \phi+A_{2} \cos 2 \Delta \phi$ is shown with a solid line. The scalar cross section is in red, and the best fit is shown with a dotted line.

Appropriately normalized to the constant term, we find the scalar $A_{2} / A_{0}$ is 0.22 , while in the spinor example, $A_{2} / A_{0}=-0.14$. Without background, the statistical significance can be estimated as $\sim\left|A_{2} / A_{0}\right| \sqrt{N}$, necessitating $\sim 30 \mathrm{fb}^{-1}$ of luminosity for measurement at several sigma. We find that the dominant partonic subprocess involves gluon radiation from light quarks. We also observe that the $\Delta \phi$ signal disappears when the pseudo-rapidity cuts are removed, indicating the dependence of this method on kinematically selecting a region in which the color-singlet VBF process is relatively enhanced.

The correlation between spin and the sign of $A_{2}$ is independent of the charge conjugation of the produced fermions. In the case of supersymmetry, for example, one may wish to determine the spin of strongly interacting superpartners (squarks and gluinos) using GF. In this case the gluinos are Majorana fermions, in contrast to the Dirac R-hadron fermions explored above. The results of an analogous simulation for $550 \mathrm{GeV}$ up-type squarks and $600 \mathrm{GeV}$ gluinos are given in figure 5 . Here we again see the sign change of the $\cos 2 \Delta \phi$ term when comparing squark $\left(A_{2} / A_{0}=0.24\right)$ and gluino $\left(A_{2} / A_{0}=-0.09\right)$ pair production. In contrast to the $R$-hadron case, isolating the signal associated with pair production of superpartners will require additional cuts to suppress Standard Model backgrounds. As our focus here is on the establishing the basic signal (and indeed, we have not specified the branching ratios of the squarks and gluinos) rather than on its isolation, we defer a detailed study of background reduction to a future analysis. We do note that previous studies of Higgs production via VBF found that the Standard Model background did not have a $2 \Delta \phi$ mode [10-12].

To ensure that the effect is not an artifact of our choice of event generator, we have repeated the calculation for the dominant partonic sub-process using Calchep [28] and confirmed the MadGraph/MadEvent results. As a final check we have computed Standard Model $t \bar{t}+j j$ production in GF kinematics and find a negative sign for $A_{2}$. Depending on the heavy particle mass $m$ and assuming decays involving heavy flavor, $t \bar{t}+j j$ could become a significant background that would mimic the $\Delta \phi$ signal associated with new fermions. In such scenarios, implementing a $b$-jet veto may be used to reduce this background $[11,12]$. 


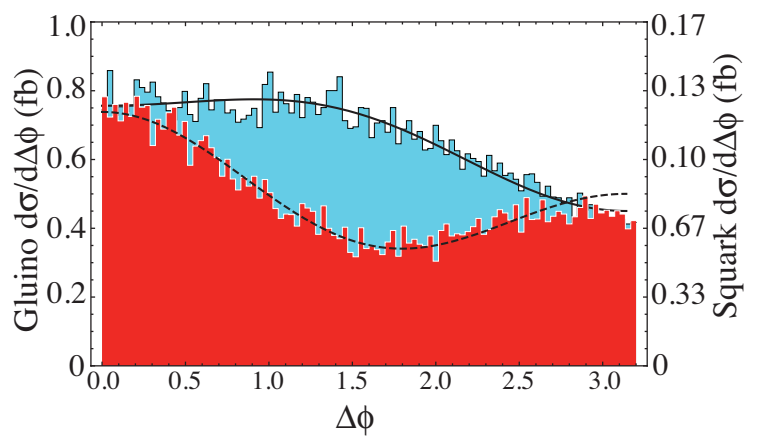

Figure 5. Differential cross section $d \sigma / d \Delta \phi$ as a function of $\Delta \phi$. The differential cross section for gluino pair production is shown in blue (scale on left), and the best fit with a solid line. The right-handed up-type squark cross section is in red (scale to right), and the best fit is shown with a dotted line.

\section{Conclusion}

In this work, we have demonstrated a proof of principle for the use of the sub-leading $\Delta \phi$ distribution as a spin diagnostic in a kinematic regime where color-singlet GF appears to be kinematically enhanced and where the heavy particle pair production is dominated by their lowest order gauge interactions. We anticipate that the method will generalize to higher spin states and to pair production through weak vector boson fusion. Strong production of $R$-hadrons provides the cleanest illustration as the signal has a large cross section and is essentially background free. Other cases - such as VBF production of supersymmetric particles - in which the heavy particle decays produce additional jets and/or missing energy will require additional cuts for appropriate signal isolation. As indicated above, recent investigations in this direction $[16,17]$ are encouraging for the prospects of appropriate jet identification in the context of GF.

More generally, one may ask whether one expects a sub-leading $\Delta \phi$-dependence associated with other multiparticle production mechanisms, such as VBF-pair production described by higher-dimension ( non-renormalizable) operators, or even particle production not associated with fusing vector bosons. As indicated earlier, we expect that the $\Delta \phi$ signal generated by VBF of an $s$-channel resonance that subsequently decays into a pair of new particles would be governed by the coupling of the fusing vector bosons to the resonance rather than by the spin of its decay products. A detailed study of these questions - along with an investigation of background suppression and other practical considerations - will appear in forthcoming work.

\section{Acknowledgments}

We would like to thank the Aspen Center for Physics where a substantial portion of this work was carried out. We also thank J. Alwall, T. Han, M. Herndon, B. Mellado, D. Morrissey, T. Plehn, W. Smith, M. Spiropulu, J. Thaler, and L.-T. Wang for helpful discussions and N. Christensen for assistance with the Calchep package. This work was supported in 
part under Department of Energy contracts DE-FG03-92-ER40701 (MRB) and DE-FG0208ER41531(MJRM) and by the Wisconsin Alumni Research Foundation (MRJM).

Open Access. This article is distributed under the terms of the Creative Commons Attribution Noncommercial License which permits any noncommercial use, distribution, and reproduction in any medium, provided the original author(s) and source are credited.

\section{References}

[1] H.-C. Cheng, K.T. Matchev and M. Schmaltz, Bosonic supersymmetry? Getting fooled at the CERN LHC, Phys. Rev. D 66 (2002) 056006 [hep-ph/0205314] [SPIRES].

[2] A.J. Barr, Using lepton charge asymmetry to investigate the spin of supersymmetric particles at the LHC, Phys. Lett. B 596 (2004) 205 [hep-ph/0405052] [SPIRES].

[3] A.J. Barr, Measuring slepton spin at the LHC, JHEP 02 (2006) 042 [hep-ph/0511115] [SPIRES].

[4] M. Battaglia, A. Datta, A. De Roeck, K. Kong and K.T. Matchev, Contrasting supersymmetry and universal extra dimensions at the CLIC multi-TeV $e^{+} e^{-}$collider, JHEP 07 (2005) 033 [hep-ph/0502041] [SPIRES].

[5] J.M. Smillie and B.R. Webber, Distinguishing spins in supersymmetric and universal extra dimension models at the Large Hadron Collider, JHEP 10 (2005) 069 [hep-ph/0507170] [SPIRES].

[6] L.-T. Wang and I. Yavin, Spin measurements in cascade decays at the LHC, JHEP 04 (2007) 032 [hep-ph/0605296] [SPIRES].

[7] A. Alves and O. Eboli, Unravelling the sbottom spin at the CERN LHC, Phys. Rev. D 75 (2007) 115013 [arXiv: 0704.0254] [SPIRES].

[8] M.R. Buckley, B. Heinemann, W. Klemm and H. Murayama, Quantum interference effects among helicities at LEP-II and Tevatron, Phys. Rev. D 77 (2008) 113017 [arXiv: 0804.0476] [SPIRES].

[9] O.J.P. Eboli and D. Zeppenfeld, Observing an invisible Higgs boson, Phys. Lett. B 495 (2000) 147 [hep-ph/0009158] [SPIRES].

[10] T. Plehn, D.L. Rainwater and D. Zeppenfeld, Determining the structure of Higgs couplings at the LHC, Phys. Rev. Lett. 88 (2002) 051801 [hep-ph/0105325] [SPIRES].

[11] V. Hankele, G. Klamke and D. Zeppenfeld, Higgs +2 jets as a probe for CP properties, hep-ph/0605117 [SPIRES].

[12] G. Klamke and D. Zeppenfeld, Higgs plus two jet production via gluon fusion as a signal at the CERN LHC, JHEP 04 (2007) 052 [hep-ph/0703202] [SPIRES].

[13] K. Hagiwara, Q. Li and K. Mawatari, Jet angular correlation in vector-boson fusion processes at hadron colliders, JHEP 07 (2009) 101 [arXiv: 0905.4314] [SPIRES].

[14] ATLAS collaboration, G. Aad et al., Expected performance of the ATLAS experiment detector, trigger and physics, arXiv:0901.0512 [SPIRES].

[15] J.D. Bjorken, How black is a constituent quark?, Acta Phys. Polon. B 23 (1992) 637 [SPIRES]. 
[16] J. Alwall, K. Hiramatsu, M.M. Nojiri and Y. Shimizu, Novel reconstruction technique for new physics processes with initial state radiation, Phys. Rev. Lett. 103 (2009) 151802 [arXiv:0905.1201] [SPIRES].

[17] M.M. Nojiri and K. Sakurai, Controlling ISR in sparticle mass reconstruction, Phys. Rev. D 82 (2010) 115026 [arXiv:1008.1813] [SPIRES].

[18] D. Krohn, L. Randall and L.-T. Wang, On the feasibility and utility of ISR tagging, arXiv: 1101.0810 [SPIRES].

[19] G.R. Farrar and P. Fayet, Bounds on R-hadron production from calorimetry experiments, Phys. Lett. B 79 (1978) 442 [SPIRES].

[20] G.R. Farrar and P. Fayet, Phenomenology of the production, decay and detection of new hadronic states associated with supersymmetry, Phys. Lett. B 76 (1978) 575 [SPIRES].

[21] B.C. Allanach, C.M. Harris, M.A. Parker, P. Richardson and B.R. Webber, Detecting exotic heavy leptons at the Large Hadron Collider, JHEP 08 (2001) 051 [hep-ph/0108097] [SPIRES].

[22] M.R. Buckley, B. Echenard, D. Kahawala and L. Randall, Stable colored particles R-SUSY relics or not?, JHEP 01 (2011) 013 [arXiv:1008.2756] [SPIRES].

[23] V.D. Barger, K.-M. Cheung, T. Han and R.J.N. Phillips, Strong $W^{+} W^{+}$scattering signals at pp supercolliders, Phys. Rev. D 42 (1990) 3052 [SPIRES].

[24] V.D. Barger, K.-M. Cheung, T. Han and D. Zeppenfeld, Single forward jet tagging and central jet vetoing to identify the leptonic $W W$ decay mode of a heavy Higgs boson, Phys. Rev. D 44 (1991) 2701 [Erratum ibid. D 48 (1993) 5444] [SPIRES].

[25] V.D. Barger, R.J.N. Phillips and D. Zeppenfeld, Mini-jet veto: a tool for the heavy Higgs search at the LHC, Phys. Lett. B 346 (1995) 106 [hep-ph/9412276] [SPIRES].

[26] D.L. Rainwater and D. Zeppenfeld, Observing $H \rightarrow W^{(*)} W^{(*)} \rightarrow e^{ \pm} \mu^{\mp} / p_{T}$ in weak boson fusion with dual forward jet tagging at the CERN LHC, Phys. Rev. D 60 (1999) 113004 [Erratum ibid. D 61 (2000) 099901] [hep-ph/9906218] [SPIRES].

[27] J. Alwall et al., MadGraph/MadEvent v4: the new web generation, JHEP 09 (2007) 028 [arXiv:0706.2334] [SPIRES].

[28] A. Pukhov et al., CompHEP: a package for evaluation of Feynman diagrams and integration over multi-particle phase space. User's manual for version 33, hep-ph/9908288 [SPIRES].

[29] M. Fairbairn et al., Stable massive particles at colliders, Phys. Rept. 438 (2007) 1 [hep-ph/0611040] [SPIRES].

[30] M. Drees and X. Tata, Signals for heavy exotics at hadron colliders and supercolliders, Phys. Lett. B 252 (1990) 695 [SPIRES].

[31] ATLAS collaboration, Background studies to searches for long-lived stopped particles decaying out-of-time with lhc collisions, ATLAS-CONF-2010-071 note, CERN, Geneva Switzerland (2010).

[32] A. Arvanitaki, S. Dimopoulos, A. Pierce, S. Rajendran and J.G. Wacker, Stopping gluinos, Phys. Rev. D 76 (2007) 055007 [hep-ph/0506242] [SPIRES].

[33] H. Baer, K.-M. Cheung and J.F. Gunion, A heavy gluino as the lightest supersymmetric particle, Phys. Rev. D 59 (1999) 075002 [hep-ph/9806361] [SPIRES]. 
[34] A. Mafi and S. Raby, An analysis of a heavy gluino LSP at CDF: the heavy gluino window, Phys. Rev. D 62 (2000) 035003 [hep-ph/9912436] [SPIRES].

[35] A. Mafi and S. Raby, A solution to the mu problem in the presence of a heavy gluino LSP, Phys. Rev. D 63 (2001) 055010 [hep-ph/0009202] [SPIRES].

[36] S. Raby, Gauge-mediated SUSY breaking with a gluino LSP, Phys. Lett. B 422 (1998) 158 [hep-ph/9712254] [SPIRES].

[37] S. Raby, Gauge mediated SUSY breaking at an intermediate scale, Phys. Rev. D 56 (1997) 2852 [hep-ph/9702299] [SPIRES].

[38] H.K. Dreiner, An introduction to explicit R-parity violation, hep-ph/9707435 [SPIRES].

[39] E.L. Berger and Z. Sullivan, Lower limits on R-parity violating couplings in supersymmetry, Phys. Rev. Lett. 92 (2004) 201801 [hep-ph/0310001] [SPIRES].

[40] ALEPH collaboration, A. Heister et al., Search for scalar quarks in $e^{+} e^{-}$collisions at $\sqrt{s}$ up to 209 GeV, Phys. Lett. B 537 (2002) 5 [hep-ex/0204036] [SPIRES].

[41] CDF collaboration, D. Acosta et al., Search for the supersymmetric partner of the top quark in dilepton events from $p \bar{p}$ collisions at $\sqrt{s}=1.8 \mathrm{TeV}$, Phys. Rev. Lett. 90 (2003) 251801 [hep-ex/0302009] [SPIRES].

[42] ALEPH, DELPHI, L3 and OPAL collaborations, R. Alemany et al., LEP2 SUSY Working Group: combined LEP GMSB Stau/Smuon/Selectron results, 189-208 GeV, CERN, Geneva Switzerland (2002).

[43] ATLAS collaboration, P. Mermod, Discovery potential of R-hadrons with the ATLAS detector at the LHC, AIP Conf. Proc. 1200 (2010) 750 [arXiv:0909.1911] [SPIRES].

[44] M. Ciccolini, A. Denner and S. Dittmaier, Strong and electroweak corrections to the production of Higgs +2 jets via weak interactions at the LHC, Phys. Rev. Lett. 99 (2007) 161803 [arXiv: 0707.0381 ] [SPIRES].

[45] M. Ciccolini, A. Denner and S. Dittmaier, Electroweak and QCD corrections to Higgs production via vector-boson fusion at the LHC, Phys. Rev. D 77 (2008) 013002 [arXiv:0710.4749] [SPIRES]. 